\title{
COMMITTEES AND SPONSORS
}

\section{Sponsoring IAU Commissions}

33 Structure and Dynamics of the Galactic System

28 Galaxies

34 Interstellar Matter

41 History of Astronomy

Commission representatives on the Scientific Organizing Committee are identified below.

\section{Scientific Organizing Committee}

A. Blaauw, Norg, Netherlands

W.B. Burton, Leiden, Netherlands (33)

C. Cesarsky, Saclay/Paris, France

E. Dekker, Boerhaave Museum, Leiden, Netherlands

J. Einasto, Tôravere, Estonia, USSR

M.W. Feast, Capetown, South Africa

K.C. Freeman, Canberra, Australia

M.A. Hoskin, Cambridge, UK (41)

D. Lynden-Be11, Cambridge, UK

M. Peimbert, lexico D.F., Mexico (34)

V. Radhakrishnan, Bangalore, India (34)

T.S. van Albada, Groningen, Netherlands

B. Westerlund, Uppsala, Sweden (28)

R. Wielen, Berlin, F.R. Germany (33)

C.G. Wynn-Williams, Honolulu, Hawaii, USA

H. van Woerden, Groningen, Netherlands, Chairman

Local Organizing Committee

R.J. Allen, Chairman

W.J. Bosman-Noteboom
J.A. de Boer, Treasurer

M. de Vries

Symposium Secretariat

J.H.M. Nunnink

G.G.A. Rouwé

J. Renner

M. van der Laan

\section{Supporting Organizations}

International Astronomical Union

University of Groningen

Koninklijke Nederlandse Akademie van Wetenschappen

Leids Kerkhoven-Bosscha Fonds

Sterrenkundig Studiefonds "Kapteyn"

Groninger Universiteits Fonds 